\title{
WHO WILL GO GREEN? A PRELIMINARY STUDY OF U.S. CONSUMER SEGMENTATION DEMOGRAPHICS
}

\author{
Siyuan Wei, Hofstra University, Hempstead, New York, USA \\ John M. Planchon, Rhodes College, Memphis, Tennessee, USA \\ William L. James, Hofstra University, Hempstead, New York, USA
}

dx.doi.org/10.18374//JBS-13-4.11

\begin{abstract}
Increased global concern over the environment has resulted in many environment-related aspects of marketing being used to address the concern. More eco-friendly products, product delivery channels, and marking communication exhorting consumers to be more eco-friendly can be seen across the globe. Given these ecological concerns, marketers are eager to identify market segments and target their marketing efforts to consumers who are more likely to respond favorably to their marketing strategies. Using a sample of 24,463 adult U.S. respondents, this study examined the demographics of gender, race, income, education, occupation, household children and age as potential segmentation variables. White or Asian, higher educated, professionals without children were found to more willing to purchase eco-friendly products and to desire to be perceived as eco-friendly consumers. These results imply that standardized segmentation techniques might not be effective globally.
\end{abstract}

Keywords: Environmental Market Segmentation; Eco-friendly Marketing; Demographic Segmentation 